
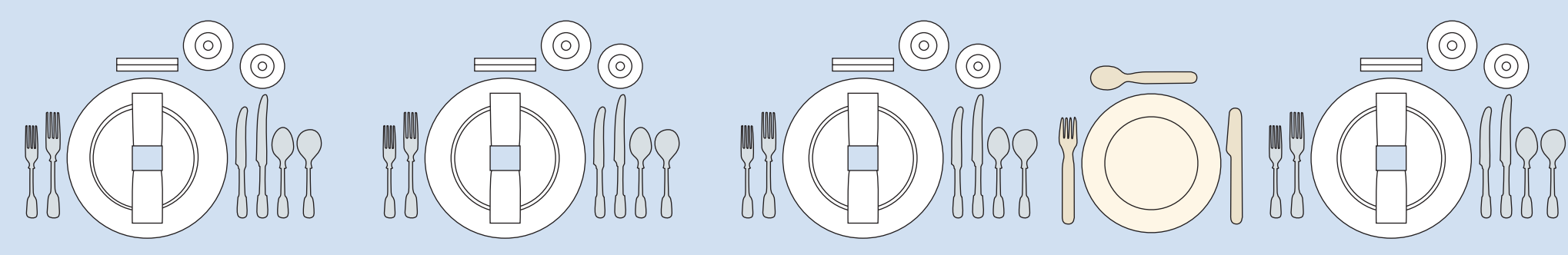

INNATE IMMUNITY

\title{
An unexpected guest at the regulatory table
}

Neutrophils are rapidly recruited to sites of inflammation and have long been regarded as the immune system's first line of defence. Now, Zhang et al. report that neutrophils also have an important regulatory role during microbial infection by secreting the anti-inflammatory cytokine interleukin-10 (ㄴ-10) in response to pattern recognition receptor (PRR) stimulation.

Previous work reported that activation of neutrophils through PRRs resulted in the production of classical pro-inflammatory cytokines. Many of these earlier studies identified neutrophils by GR1 expression; however, this antigen is found on other cell types, including inflammatory monocytes. By distinguishing between true neutrophils and GR1 ${ }^{+}$ monocytes with neutrophil-selective LY6G-specific antibodies, the authors found that monocytes, but not neutrophils, produced pro-inflammatory cytokines and chemokines following stimulation with mycobacteria. Surprisingly, neutrophils secreted IL-10 in response to bacterial stimulation. Next, the authors addressed the signalling pathways involved in neutrophil induction of IL-10. Using gene-deficient mice, they found that Toll-like receptor 2 (TLR2) and the TLR adaptor myeloid differentiation primaryresponse protein 88 (MYD88) were required for neutrophil production of IL-10. However, synthetic TLR2 agonists could not induce the IL-10 response induced by whole bacteria, suggesting that co-activation of both TLRs and other PRRs is necessary for neutrophils to make IL-10. The authors identified the other key PRRs involved as members of the C-type lectin receptor family (CLRs). Although activation of either TLRs or CLRs alone did not result in marked neutrophil production of IL-10, co-ligation of these receptors led to substantial IL-10 production by neutrophils. Using loss-of-function mutants and pharmacological inhibitors, the authors showed that neutrophil generation of IL-10 involved co-activation of MYD88 and the CLR signalling adaptor spleen tyrosine kinase (SYK). Notably, these signalling pathways are normally associated with driving pro-inflammatory cytokine production by monocytes and macrophages.

To extend their findings, the authors depleted neutrophils with LY6G-specific antibodies, before challenging the mice intranasally with Mycobacterium bovis. Cells from alveolar washes of the infected neutrophil-depleted mice produced less IL-10 and higher levels of tumour necrosis factor than cells from control animals; however, equivalent levels of bacteria were found in lung tissue from both groups. One explanation for the similar mycobacterial loads, despite the loss of bacterial-killing activity by neutrophils, was the increased levels of pro-inflammatory cytokines in the lungs caused by neutrophil depletion. To test this, the authors removed the anti-inflammatory potential of neutrophils by conducting similar lung infection experiments in IL-10deficient mice. In keeping with their hypothesis, IL-10-deficient mice that were depleted of neutrophils prior to lung infection were found to have greater bacterial loads than neutrophil-competent IL-10deficient controls. Furthermore, in transfer experiments, in which mice received either wild-type or IL-10deficient neutrophils, the recipients of the IL-10-deficient neutrophils had lower bacterial loads in the lung than the recipients of the wild-type neutrophils.

Taken together, the data show a previously unidentified regulatory role for neutrophils and suggest that this innate cell population may have a role of dual importance during host infection: engulfing and eliminating pathogenic bacteria while dampening tissue inflammation through the production of IL- 10 .

Yvonne Bordon

ORIGINAL RESEARCH PAPER Zhang, X. et al. Coactivation of Syk kinase and MyD88 adaptor protein pathways by bacteria promotes regulatory properties of neutrophils. Immunity 31, 761-771 (2009) 